\title{
ON MAXIMAL ESSENTIAL EXTENSIONS OF RINGS
}

\author{
R. R. ANDRUSZKIEWICZ
}

(Received 25 June 2010)

\begin{abstract}
The main purpose of this paper is to give a new, elementary proof of Flanigan's theorem, which says that a given ring $A$ has a maximal essential extension $\operatorname{ME}(A)$ if and only if the two-sided annihilator of $A$ is zero. Moreover, we discuss the problem of description of $\operatorname{ME}(A)$ for a given right ideal $A$ of a ring with an identity.
\end{abstract}

2010 Mathematics subject classification: primary 16D25; secondary 16 S70.

Keywords and phrases: ideal, essential ideal, essential extension, module.

\section{Introduction}

All rings in this paper are associative but we do not assume that each ring has an identity element. To denote that $I$ is an ideal (respectively a left ideal, a right ideal) of a ring $R$, we write $I \triangleleft R$ (respectively $I<_{l} R, I<_{r} R$ ). We say that a subring $A$ of a ring $R$ is essential in $R$ or that $R$ is an essential extension of $A$, if $A \cap I \neq 0$ for every nonzero ideal $I$ of $R$.

The idealizer $\operatorname{Id}_{R}(A)$ of a subring $A$ of a ring $R$ is the largest subring of $R$ in which $A$ is an ideal, that is, $\operatorname{Id}_{R}(A)=\{r \in R: r A \subseteq A$ and $A r \subseteq A\}$. For a nonempty subset $X$ of a ring $R$ the left annihilator of $X$ is $l_{R}(X)=\{r \in R: r X=0\}$ and the right annihilator of $X$ is $r_{R}(X)=\{r \in R: X r=0\}$. The two-sided annihilator of $X$ is defined to be the subring $a_{R}(X)=l_{R}(X) \cap r_{R}(X)$. To simplify the notation we write $a(R)$ instead of $a_{R}(R)$.

In [2] Beidar introduced the notion of a maximal essential extension of a ring.

DEFINITION 1.1 (Beidar). A ring $R$ is said to be a maximal essential extension of a ring $A(R=\operatorname{ME}(A))$, if $A$ is an essential ideal of $R$ and, for any ring $S$ which contains $A$ as an ideal, there exists a ring homomorphism $h: S \rightarrow R$ such that $h(a)=a$ for all $a \in A$.

There are many important applications of maximal essential extension of rings (see [5, 6]), especially in the theory of radicals. We refer the reader to [2] for a thorough discussion of the various applications of this idea in solving significant

(C) 2010 Australian Mathematical Publishing Association Inc. 0004-9727/2010 \$16.00 
problems in the theory of rings. In [2] Beidar asked when a given ring $A$ has $\operatorname{ME}(A)$. It turned out that an answer this question was already given in [3] by Flanigan. However, the author used advanced methods and techniques of category theory. The Flanigan theorem can be presented in the following form.

THEOREM 1.2 (Flanigan). A ring $A$ has $\operatorname{ME}(A)$ if and only if $a(A)=\{0\}$.

The main purpose of this paper is to give a new elementary proof of Flanigan's theorem (see Section 3). Moreover, we present an example which shows that in [2, Theorem 6], given without a proof, there are few assumptions missing. For the proof the author refers to [1], but that paper only gives a proof of a special case of the theorem under discussion. We give necessary assumptions for [2, Theorem 6] and show that they cannot be weakened.

\section{Preliminaries}

For any element $a$ of a ring $R$ we denote the ideal of $R$ generated by $a$ by $R^{1} a R^{1}$. We denote the additive group of $A$ by $A^{+}$and we denote the ring of endomorphisms of the right (respectively left) $A$-module $A$ by $\operatorname{End}\left(A_{A}\right)$ (respectively $\operatorname{End}\left({ }_{A} A\right)$ ). For ${ }_{A} A$ we write endomorphisms on the right of the arguments and consequently we will use the right-hand rule for composition of mappings. The $\operatorname{ring} \operatorname{End}\left(A_{A}\right) \oplus \operatorname{End}\left({ }_{A} A\right)$ will be denoted by $E(A)$. It is easy to check that

$$
\Omega(A)=\left\{(\lambda, \rho) \in E(A): a \cdot \lambda(b)=(a) \rho \cdot b \forall_{a, b \in A}\right\}
$$

is a subring of $E(A)$. If $A \triangleleft S$ then for $s \in S$ we define $l_{s}: A \rightarrow A$ by $l_{S}(a)=s \cdot a$ and $r_{s}: A \rightarrow A$ by $(a) r_{s}=a \cdot s$. A trivial verification shows that $l_{s} \in \operatorname{End}\left(A_{A}\right)$, $r_{s} \in \operatorname{End}\left({ }_{A} A\right)$ and $\left(l_{s}, r_{s}\right) \in \Omega(A)$ for any $s \in S$. Moreover, the map $f: S \rightarrow \Omega(A)$ given by $f(s)=\left(l_{s}, r_{s}\right)$ for $s \in S$ is a ring homomorphism such that $\operatorname{Ker} f=a_{S}(A)$. In particular, for $S=A$ we obtain that $\bar{A}=\left\{\left(l_{a}, r_{a}\right): a \in A\right\}$ is a subring of $\Omega(A)$. It is seen at once that, for all $a \in A$ and $(\lambda, \rho) \in \Omega(A)$, we have $(\lambda, \rho) \cdot\left(l_{a}, r_{a}\right)=$ $\left(l_{\lambda(a)}, r_{\lambda(a)}\right)$ and $\left(l_{a}, r_{a}\right) \cdot(\lambda, \rho)=\left(l_{(a) \rho}, r_{(a) \rho}\right)$. It follows that $\bar{A} \triangleleft \Omega(A)$. If, in addition, $a(A)=\{0\}$, then $\bar{A} \cong A$.

PROposition 2.1. If $A$ is an ideal of a ring $R$ and $a(A)=\{0\}$ then $A$ is an essential ideal in $R$ if and only if $a_{R}(A)=\{0\}$.

Proof. If $a_{R}(A)=\{0\}$ and $J$ is a nonzero ideal of a ring $R$ then $J A \neq\{0\}$ or $A J \neq\{0\}$ and $J A \cup A J \subseteq A \cap J$. Thus $A \cap J \neq\{0\}$. Hence $A$ is an essential ideal of $R$. Conversely, assume that $A$ is an essential ideal of $R$. Since $a_{R}(A) \triangleleft R$ and $a_{R}(A) \cap A=a(A)$, it follows that $a_{R}(A)=\{0\}$.

Proposition 2.2. Let $A$ be a ring such that $a(A)=\{0\}$. Assume that $A$ is an essential ideal of a ring $R$ and $A$ is a subring of a ring $S$ such that $a_{S}(A)=\{0\}$. Then:

(i) every ring homomorphism $f: R \rightarrow S$ such that $f(a)=$ a for every $a \in A$ is an embedding;

(ii) there exists at most one homomorphism $f: R \rightarrow S$ such that $f(a)=$ a for all $a \in A$. 
Proof. (i) Assume that the homomorphism $f: R \rightarrow S$ is not an embedding. Then Ker $f \neq\{0\}$, so Ker $f \cap A \neq\{0\}$. Hence there exists $0 \neq a \in A$ such that $f(a)=0$. But $a=f(a)$, which is a contradiction.

(ii) Suppose that $f$ and $g$ are homomorphisms of $R$ into $S$ such that $\left.f\right|_{A}=\mathrm{id}_{A}$ and $\left.g\right|_{A}=\operatorname{id}_{A}$. Let $x \in R$ and $a \in A$. Then

$$
\begin{aligned}
(f(x)-g(x)) a & =f(x) a-g(x) a=f(x) f(a)-g(x) g(a) \\
& =f(x a)-g(x a)=x a-x a=0,
\end{aligned}
$$

since $x a \in A$. So $(f(x)-g(x)) A=\{0\}$. Similarly, one can prove that $A(f(x)-$ $g(x))=\{0\}$. From this we conclude that $f(x)-g(x) \in a_{S}(A)$. But $a_{S}(A)=\{0\}$, so $f(x)=g(x)$ for every $x \in R$. This shows that $f=g$.

Proposition 2.3. Let $A$ be a ring such that $a(A)=\{0\}$. Then $a_{E(A)}(\bar{A})=\{0\}$ and $\Omega(A)=\operatorname{id}_{E(A)}(\bar{A})$. Moreover, if $A$ is an essential ideal of a ring $S$ then the function $s \mapsto\left(l_{s}, r_{s}\right)$ for $s \in S$ is the unique ring homomorphism of $S$ into $\Omega(A)$ such that $a \mapsto\left(l_{a}, r_{a}\right)$ for $a \in A$.

PROOF. Let $(f, g) \in a_{E(A)}(\bar{A})$. Then for any $a \in A$ we have $(f, g) \cdot\left(l_{a}, l_{r}\right)=(0,0)$ and $\left(l_{a}, r_{a}\right) \cdot(f, g)=(0,0)$, so that $f l_{a}=0, l_{a} f=0, g r_{a}=0$ and $r_{a} g=0$. Hence, for every $b \in A$ we have

$$
\begin{gathered}
0=\left(f l_{a}\right)(b)=f(a \cdot b)=f(a) \cdot b, \quad 0=\left(l_{b} f\right)(a)=b \cdot f(a), \\
0=(b)\left(g r_{a}\right)=(b) g \cdot a \quad \text { and } \quad 0=(a)\left(r_{b} g\right)=(a \cdot b) g=a \cdot(b) g .
\end{gathered}
$$

This means that $f(a),(b) g \in a(A)=\{0\}$, hence $f=0, g=0$ and $a_{E(A)}(\bar{A})=\{0\}$.

Recall that $\bar{A} \triangleleft \Omega(A)$ implies that $\Omega(A) \subseteq \operatorname{Id}_{E(A)}(\bar{A})$. Moreover, $\bar{A} \triangleleft \operatorname{Id}_{E(A)}(\bar{A})$ and $a_{E(A)}(\bar{A})=\{0\}$ and $\bar{A} \cong A$, so Proposition 2.1 gives that $\bar{A}$ is an essential ideal of $\operatorname{Id}_{E(A)}(\bar{A})$ and there exists an embedding of rings $h: \operatorname{Id}_{E(A)}(\bar{A}) \rightarrow \Omega(A)$ such that $\left.h\right|_{\bar{A}}=\mathrm{id}_{\bar{A}}$. By Propositions 2.1 and 2.2, we get that the identity mapping on $\operatorname{Id}_{E(A)}(\bar{A})$ is the unique ring homomorphism of $\operatorname{Id}_{E(A)}(\bar{A})$ into $E(A)$ which is an identity map on $\bar{A}$. Hence $h\left(\operatorname{Id}_{E(A)}(\bar{A})\right)=\operatorname{Id}_{E(A)}(\bar{A})$ implies that $\operatorname{Id}_{E(A)}(\bar{A}) \subseteq \Omega(A)$ and $\operatorname{Id}_{E(A)}(\bar{A})=\Omega(A)$.

Finally, suppose that $A$ is an essential ideal of $S$. Applying Proposition 2.2, we get that the function $g: S \rightarrow \Omega(A)$ given by $g(s)=\left(l_{s}, r_{s}\right)$ for $s \in S$ is the unique ring homomorphism such that $g(a)=\left(l_{a}, r_{a}\right)$ for $a \in A$.

\section{Proof of the Flanigan theorem}

A few facts in this section are well known, but we will prove them for completeness.

LEMMA 3.1. Let $A, B$ be nonzero rings such that $a(B)$ is an essential ideal of $B$ and $f: a(A) \rightarrow a(B)$ is an isomorphism of rings. Then

$$
\begin{gathered}
I=\{(a, f(a)): a \in a(A)\} \triangleleft A \times B, \\
{[(A \times\{0\})+I] / I \cong A, \quad[(\{0\} \times B)+I] / I \cong B \quad \text { and } \quad[(A \times\{0\})+I] / I}
\end{gathered}
$$

is an essential ideal of the ring $(A \times B) / I$. 
Proof. Directly from the assumptions we get that $I$ is a subgroup of $(A \times B)^{+}$and $I \subseteq a(A \times B)$. Hence, in particular, $I \triangleleft A \times B$. Moreover,

$$
(A \times\{0\}) \cap I=\{(a, f(a)): a \in a(A), f(a)=0\}=\{(0,0)\},
$$

so $[(A \times\{0\})+I] / I \cong A$. Next,

$$
(\{0\} \times B) \cap I=\{(a, f(a)): a=0\}=\{(0,0)\}
$$

implies that $[(\{0\} \times B)+I] / I \cong B$.

Suppose that $J \triangleleft A \times B$ and $I \subset J$. Recall that it is sufficient to prove that

$$
[(A \times\{0\})+I] / I \cap J / I \neq 0 .
$$

There exists $(a, b) \in A \times B$ such that $(a, b) \in J \backslash I$. If $a \notin a(A)$ then there exists $c \in A$ such that $c a \neq 0$ or $a c \neq 0$. If $c a \neq 0$ then

$$
I \neq(c a, 0)+I=[(c, 0)+I] \cdot[(a, b)+I] \in[(A \times\{0\})+I] / I \cap J / I .
$$

Likewise, if $a c \neq 0$ then

$$
I \neq(a c, 0)+I=[(a, b)+I] \cdot[(c, 0)+I] \in[(A \times\{0\})+I] / I \cap J / I .
$$

Assume that $a \in a(A)$. Then $I \neq(a, b)+I=(0, b-f(a))+I$. Consequently $0 \neq b-f(a) \in B$. Essentiality of $a(B)$ implies that there exists

$$
0 \neq y \in\left[B^{1}(b-f(a)) B^{1}\right] \cap a(B) .
$$

Then $(0, y)+I \in J$. But $f$ is onto, so $y=f(x)$ for some $x \in a(A)$. Notice that $x \neq 0$, because $y \neq 0$. Thus

$$
\begin{aligned}
I & \neq(0, y)+I=[(x, f(x))+(-x, 0)]+I \\
& =(-x, 0)+I \in[(A \times\{0\})+I] / I \cap J / I .
\end{aligned}
$$

This concludes the proof.

LEMMA 3.2. Let $M^{+}$be a nonzero abelian group and $T$ be a nonempty set. Denote $M_{t}=M$ for $t \in T$ and $N=M \oplus \bigoplus_{t \in T} M_{t}, S=\{f \in \operatorname{End}(N): f(M)=0\}$. Then $S<_{l} \operatorname{End}(N)$ and $\left[\begin{array}{ll}0 & M \\ 0 & 0\end{array}\right]=a(B)$ is an essential ideal of the ring $B=\left[\begin{array}{ll}S & N \\ 0 & 0\end{array}\right]$.

PROOF. An easy computation shows that $S<_{l} \operatorname{End}(N)$ and

$$
\left[\begin{array}{cc}
0 & M \\
0 & 0
\end{array}\right] \subseteq a(B) \text {. }
$$

Let

$$
\left[\begin{array}{cc}
f_{0} & x_{0} \\
0 & 0
\end{array}\right] \in a(B) .
$$

Suppose that $f_{0} \neq 0$. Then there exists $y \in N$ such that $f_{0}(y) \neq 0$. Hence

$$
\left[\begin{array}{cc}
f_{0} & x_{0} \\
0 & 0
\end{array}\right] \cdot\left[\begin{array}{ll}
0 & y \\
0 & 0
\end{array}\right]=\left[\begin{array}{cc}
0 & f_{0}(y) \\
0 & 0
\end{array}\right] \neq\left[\begin{array}{ll}
0 & 0 \\
0 & 0
\end{array}\right],
$$

which is a contradiction. Thus $f_{0}=0$. Assume that $x_{0} \notin M$. For $t \in T$ let $\pi_{t}$ denote a projection of $N$ onto $M_{t}$. It is clear that $\pi_{t} \in S$ for $t \in T$. But $x_{0} \notin M$, so there 
exists $t_{0} \in T$ such that $\pi_{t_{0}}\left(x_{0}\right) \neq 0$. Moreover,

$$
\left[\begin{array}{ll}
0 & 0 \\
0 & 0
\end{array}\right] \neq\left[\begin{array}{cc}
\pi_{t_{0}} & 0 \\
0 & 0
\end{array}\right] \cdot\left[\begin{array}{cc}
0 & x_{0} \\
0 & 0
\end{array}\right]
$$

which is impossible. Hence $x_{0} \in M$ and, finally,

$$
a(B)=\left[\begin{array}{cc}
0 & M \\
0 & 0
\end{array}\right] .
$$

Let $J$ be a nonzero ideal of $B$. Then there exists

$$
\left[\begin{array}{ll}
0 & 0 \\
0 & 0
\end{array}\right] \neq\left[\begin{array}{ll}
f & x \\
0 & 0
\end{array}\right] \in J .
$$

If $f \neq 0$ then we can take $y \in N$ such that $f(y) \neq 0$. Next,

$$
\left[\begin{array}{ll}
0 & 0 \\
0 & 0
\end{array}\right] \neq\left[\begin{array}{cc}
0 & f(y) \\
0 & 0
\end{array}\right]=\left[\begin{array}{cc}
f & x \\
0 & 0
\end{array}\right] \cdot\left[\begin{array}{ll}
0 & y \\
0 & 0
\end{array}\right] \in J .
$$

So $\left[\begin{array}{ll}0 & n \\ 0 & 0\end{array}\right] \in J$ for some $0 \neq n \in N$. If $n \in M$ then

$$
\left[\begin{array}{ll}
0 & n \\
0 & 0
\end{array}\right] \in J \cap a(B) .
$$

If $n \notin M$ then there exists $t_{0} \in T$ such that $\pi_{t_{0}}(n) \neq 0$. Let $g_{0}: M_{t_{0}} \rightarrow M$ be a natural isomorphism. Then $g_{0} \pi_{t_{0}} \in S, 0 \neq\left(g_{0} \pi_{t_{0}}\right)(n) \in M$ and

$$
\left[\begin{array}{cc}
0 & \left(g_{0} \pi_{t_{0}}\right)(n) \\
0 & 0
\end{array}\right]=\left[\begin{array}{cc}
g_{0} \pi_{t_{0}} & 0 \\
0 & 0
\end{array}\right] \cdot\left[\begin{array}{ll}
0 & n \\
0 & 0
\end{array}\right] \in J \cap a(B) .
$$

This proves that $a(B)$ is an essential ideal of $B$.

THEOREM 3.3. Let $A$ be a ring such that $a(A) \neq\{0\}$. Then for any cardinal number $\alpha$ there exists a ring $R$ such that card $R \geq \alpha$ and $A$ is an essential ideal of $R$.

ProOf. Let $T$ be a set of cardinality $\alpha \geq 1$. Let $M_{t}=a(A)$ for $t \in T$. Then $N=a(A) \oplus \bigoplus_{t \in T} M_{t}$ has cardinality greater than or equal to $\alpha$. Put

$$
S=\{f \in \operatorname{End}(N): f(a(A))=0\} .
$$

According to Lemma 3.2

$$
a(A) \cong\left[\begin{array}{cc}
0 & a(A) \\
0 & 0
\end{array}\right]=a(B) \text { for } B=\left[\begin{array}{cc}
S & N \\
0 & 0
\end{array}\right] .
$$

Moreover, $a(B)$ is an essential ideal of $B$ and card $B \geq \alpha$. The function $f: a(A) \rightarrow$ $a(B)$ defined by

$$
f(x)=\left[\begin{array}{ll}
0 & x \\
0 & 0
\end{array}\right] \quad \text { for } x \in a(A)
$$


is an isomorphism of rings. Denote $I=\{(a, f(a)): a \in a(A)\}$. By Lemma 3.1 we get that

$$
A \cong[(A \times\{0\})+I] / I \triangleleft(A \times B) / I, \quad \operatorname{card}((A \times B) / I) \geq \operatorname{card} B \geq \alpha
$$

and $[(A \times\{0\})+I] / I$ is an essential ideal of $(A \times B) / I$.

Theorem 3.4 (Flanigan). A ring $A$ has $\operatorname{ME}(A)$ if and only if $a(A)=\{0\}$. Moreover, if $a(A)=\{0\}$ then $\Omega(A)=\operatorname{ME}(A)$.

Proof. Suppose that $R=\operatorname{ME}(A)$. If $a(A) \neq\{0\}$ then by Theorem 3.3 there exists a ring $S$ of cardinality less than card $R$ which is an essential extension of $A$. Hence there exists a ring homomorphism $f: S \rightarrow R$ such that $\left.F\right|_{A}=\operatorname{id}_{A}$. Thus Ker $f \cap A=\{0\}$ and consequently Ker $f=\{0\}$ which means that $f$ is an embedding. But card $R<$ card $S$, a contradiction. Finally, $a(A)=\{0\}$.

Conversely, assume that $a(A)=\{0\}$. The mapping $a \mapsto\left(l_{a}, r_{a}\right)$ for $a \in A$ is an isomorphism of $A$ onto a ring $\bar{A}$. By Propositions 2.1 and $2.3, \bar{A}$ is an essential ideal of the ring $\Omega(A)$. Let $A$ be an ideal of $S$. Then the function $f: S \rightarrow \Omega(A)$ given by $f(s)=\left(l_{s}, r_{s}\right)$ for $s \in S$ is a ring homomorphism and $f(a)=\left(l_{a}, r_{a}\right)$ for $a \in A$. Thus $\Omega(A)=\operatorname{ME}(A)$.

Proposition 3.5. If $R=\operatorname{ME}(A)$ and $S=\operatorname{ME}(A)$ then there exists a unique ring homomorphism $f: R \rightarrow S$ such that $\left.f\right|_{A}=\mathrm{id}_{A}$ and this homomorphism is an isomorphism.

Proof. Theorem 3.4 implies that $a(A)=\{0\}$. By definition of $\operatorname{ME}(A)$ we get that there exist ring homomorphisms $f: R \rightarrow S, g: S \rightarrow R$ such that $\left.f\right|_{A}=\mathrm{id}_{A}$ and $\left.g\right|_{A}=\mathrm{id}_{A}$. Thus $g \circ f: R \rightarrow R$ and $f \circ g: S \rightarrow S$ are homomorphisms of rings such that $\left.g \circ f\right|_{A}=\mathrm{id}_{A}$ and $\left.f \circ g\right|_{A}=\mathrm{id}_{A}$. From this and Propositions 2.1 and 2.2 we conclude that $g \circ f=\mathrm{id}_{R}$ and $f \circ g=\mathrm{id}_{S}$. Hence $f$ and $g$ are isomorphisms. Moreover, applying Proposition 2.2, we see that $f$ is the unique homomorphism of $R$ into $S$.

\section{Examples and applications}

In many problems concerning the structure of rings, properties of the ring $\operatorname{ME}(A)$ play an important role, especially in the case where $\operatorname{ME}(A)$ is precisely described. In [5, Theorem 2] it was proved that if $a$ is a regular element of a ring $R$ with identity then $\operatorname{Id}_{R}(a R)=\operatorname{ME}(a R)$.

Definition 4.1 (Beidar). Let $R$ be a ring with identity and let $M$ be a right unital $R$-module. We say that $M$ is a generator if

$$
\sum\left\{f(M): f \in \operatorname{Hom}\left(M_{R}, R_{R}\right)\right\}=R,
$$

that is, there exist $f_{1}, \ldots, f_{s} \in \operatorname{Hom}\left(M_{R}, R_{R}\right)$ and $m_{1}, \ldots, m_{s} \in M$ such that

$$
1=f_{1}\left(m_{1}\right)+\cdots+f_{S}\left(m_{s}\right) \text {. }
$$

EXAMPLE 4.2.

(a) If $a \in R$ is a right regular element of a ring $R$ with an identity then the function $h: R \rightarrow a R$ given by $h(x)=a \cdot x$ is an isomorphism of a right regular $R$-module. 
Thus $h^{-1}: a R \rightarrow R$ is also an isomorphism of a right regular $R$-module and $M=a R$ is a generator.

(b) Let $J$ be a right ideal of a ring $R$ with an identity such that $R J=R$. Then there exist $j_{1}, \ldots, j_{s} \in J$ and $a_{1}, \ldots, a_{s} \in R$ such that $1=a_{1} j_{1}+\cdots+a_{s} j_{s}$, so $1=l_{a_{1}}\left(j_{1}\right)+\cdots+l_{a_{s}}\left(j_{s}\right)$. Hence, $M=J$ is a generator.

In [2, Theorem 6] Beidar stated without proof that if $I$ is a right ideal of a ring $R$ with an identity and if $I_{R}$ is a generator, then $\operatorname{Id}_{R}(I)=\operatorname{ME}(I)$. Unfortunately, this theorem does not hold in general. However, the theorem is true if we add following assumption:

for any $h \in \operatorname{Hom}\left({ }_{I} I, l_{I}(I)\right)$ there exists $c \in R$ such that $h=r_{c}$.

There are many examples of rings $R$ with an identity and $I<_{r} R$ such that $I_{R}$ is a generator, but for which condition (4.1) does not hold.

EXAMPLE 4.3. Let $S$ be a polynomial ring in two noncommuting variables $X, Y$ over the ring $\mathbb{Z}$ of integers. Let $J$ be an ideal of $S$ generated by $Y X$ and $Y^{2}$. Let $x=X+J$, $y=Y+J$ and $R=S / J$. Then

$$
R=\mathbb{Z}[x]+\mathbb{Z}[x] y .
$$

Moreover, $x$ is a right regular element of $R$. Let $I=x R$. It is obvious that $I=x \mathbb{Z}[x]+x \mathbb{Z}[x] y, I_{R}$ is a generator and $\operatorname{Id}_{R}(I)=R$. We see at once that $l_{I}(I)=$ $x \mathbb{Z}[x] y$. Let $h: I \rightarrow l_{I}(I)$ be defined by

$$
h(a+b y)=(a+b) y \quad \text { for } a, b \in x \mathbb{Z}[x] .
$$

Then $h \in \operatorname{Hom}\left({ }_{I} I, l_{I}(I)\right)$. If $h=r_{c}$ for some $c \in R$ then $h(x)=(x) r_{c}$ and consequently $x y=x c$ and $c=y$. But this and $y^{2}=0$ imply that $x y=h(x y)=$ $(x y) r_{y}=x y^{2}=0$, which is a contradiction.

THEOREM 4.4. Let $I$ be a right ideal of a ring $R$ with an identity and $I_{R}$ be a generator. Then $\operatorname{Id}_{R}(I)=\operatorname{ME}(I)$ if and only if for every $h \in \operatorname{Hom}\left({ }_{I} I, l_{I}(I)\right)$ there exists $c \in R$ such that $h=r_{c}$.

PROOF. By assumption, there exist $f_{1}, \ldots, f_{s} \in \operatorname{Hom}\left(I_{R}, R_{R}\right)$ and $i_{1}, \ldots, i_{s} \in I$ such that

$$
1=f_{1}\left(i_{1}\right)+\cdots+f_{s}\left(i_{s}\right) .
$$

From (4.2) we conclude that for any $r \in R$ we have $r=f_{1}\left(i_{1} r\right)+\cdots+f_{s}\left(i_{s} r\right)$. Therefore $r_{R}(I)=\{0\}$. Hence $a_{R}(I)=\{0\}$, so by Theorem 3.4, $\Omega(A)=\operatorname{ME}(A)$, $I$ is an essential in $R$ and, moreover, $I$ is an essential ideal of $T=\operatorname{Id}_{R}(I)$.

Suppose that $T=\operatorname{ME}(I)$. Applying Theorem 3.4 and Proposition 3.5, we see that the function $f: T \rightarrow \Omega(I)$ given by $f(t)=\left(l_{t}, r_{t}\right)$ for $t \in T$ is an isomorphism of rings. Choose any $h \in \operatorname{Hom}\left({ }_{I} I, l_{I}(I)\right)$. Then $(0, h) \in \Omega(I)$, so there exists $c \in T$ such that $(0, h)=\left(l_{c}, r_{c}\right)$ and $h=r_{c}$.

Conversely, assume that for every $h \in \operatorname{Hom}\left({ }_{I} I, l_{I}(I)\right)$ there exists $c \in R$ such that $h=r_{c}$. Then $I c \subseteq l_{I}(I)$, which implies that $I c I=\{0\}$. Hence $c I \subseteq r_{I}(I)=\{0\}$ 
and $c \in l_{I}(I)$. Therefore $c \in T$ and $(0, h)=\left(l_{c}, r_{c}\right) \in \Omega(I)$. By Propositions 2.2, 2.3 and Theorem 3.4 it suffices to prove that the function $f: T \rightarrow \Omega(I)$ given by $f(t)=\left(l_{t}, r_{t}\right)$ for $t \in T$ is onto. Fix any $(\lambda, \rho) \in \Omega(I)$. Then for all $i, j \in I$, $i \cdot \lambda(j)=(i) \rho \cdot j$. Let

$$
b=f_{1}\left(\left(i_{1}\right) \rho\right)+\cdots+f_{s}\left(\left(i_{s}\right) \rho\right) .
$$

Notice that $b \in R$ and, for any $j \in I$,

$$
\begin{aligned}
b \cdot j & =f_{1}\left(\left(i_{1}\right) \rho\right) \cdot j+\cdots+f_{s}\left(\left(i_{s}\right) \rho\right) \cdot j=f_{1}\left(\left(i_{1}\right) \rho \cdot j\right)+\cdots+f_{s}\left(\left(i_{s}\right) \rho \cdot j\right) \\
& =f_{1}\left(i_{1} \lambda(j)\right)+\cdots+f_{k}\left(i_{s} \lambda(j)\right)=f_{1}\left(i_{1}\right) \cdot \lambda(j)+\cdots+f_{s}\left(i_{s}\right) \cdot \lambda(j)=\lambda(j)
\end{aligned}
$$

by (4.1). Hence, $\lambda=l_{b}$. From this, $b \in T$. Next, for

$$
i, j \in I:(j \cdot b-(j) \rho) \cdot i=j \cdot(b \cdot i)-(j) \rho \cdot i=j \cdot \lambda(i)-j \cdot \lambda(i)=0,
$$

so $j \cdot b-(j) \rho \in l_{I}(I)$. By the above, the function $h$ defined by $h(j)=j \cdot b-(j) \rho$ for $j \in I$ is a homomorphism of the left $I$-module $I$ into the left $I$-module $l_{I}(I)$. Thus there exists $c \in l_{I}(I)$ such that $h=r_{c}$ and $\left(l_{c}, r_{c}\right)=(0, h) \in \Omega(I)$. But $h=r_{b}-\rho$ so

$$
\rho=r_{b}-h=r_{b}-r_{c}=r_{b-c}, \quad \lambda=l_{b}=l_{b}-l_{c}=l_{b-c}
$$

and, finally, $(\lambda, \rho)=\left(\lambda_{b-c}, \rho_{b-c}\right) \in \Omega(I)$.

COROLlary 4.5. Let $R$ be a ring with an identity and assume that $I<_{r} R$ is a generator. If $l_{I}(I)=\{0\}$ then $\operatorname{Id}_{R}(I)=\mathrm{ME}(I)$. In particular, if a is a two-sided regular element of $R$ then $\operatorname{Id}_{R}(a R)=\operatorname{ME}(a R)$.

Corollary 4.6 (Beidar). Let $R$ be a ring with an identity and let $I<_{r} R$ and $R I=R$. Then $\operatorname{Id}_{R}(I)=\operatorname{ME}(I)$. In particular, $\operatorname{Id}_{R}(J)=\operatorname{ME}(J)$ for every nonzero right ideal $J$ of a simple ring $R$ with an identity.

PROOF. By assumption, there exist $r_{1}, \ldots, r_{s} \in R$ and $i_{1}, \ldots, i_{s} \in I$ such that $1=$ $r_{1} i_{1}+\cdots+r_{s} i_{s}$. Hence $r_{R}(I)=\{0\}$ and $1=l_{r_{1}}\left(i_{1}\right)+\cdots+l_{r_{s}}\left(i_{s}\right)$. Thus $I_{R}$ is a generator. Choose $h \in \operatorname{Hom}\left({ }_{I} I, l_{I}(I)\right)$. Let $b=r_{1} h\left(i_{1}\right)+\cdots+r_{s} h\left(i_{s}\right)$. Then $b \in R$ and, for every $i \in I$,

$$
i=\left(i r_{1}\right) i_{1}+\cdots+\left(i r_{s}\right) i_{s} \text { and } h(i)=\left(i r_{1}\right) h\left(i_{1}\right)+\cdots+\left(i r_{s}\right) h\left(i_{s}\right)=i \cdot b .
$$

Thus $h=r_{b}$. From Theorem 4.4 it follows that $\operatorname{Id}_{R}(I)=\operatorname{ME}(I)$.

Definition 4.7. A simple ring $A=A^{2}$ satisfies a Gardner condition if, for any ring $R, A \triangleleft R$ and $R / A \cong A$ imply that there exists $I \triangleleft R$ such that $R=A \oplus I$.

PROPOSITION 4.8. A simple ring $A=A^{2}$ satisfies a Gardner condition if and only if $A$ is not embeddable in a ring $\mathrm{ME}(A) / A$.

Proof. Let $A=A^{2}$ be a simple ring. Then $a(A)=\{0\}$, so by Theorem 3.4 there exists $\operatorname{ME}(A)$. First, assume that $A$ does not satisfy a Gardner condition. Then there exists a ring $R$ in which $A \triangleleft R, R / A \cong A$ and there does not exist $I \triangleleft R$ such that $R=A \oplus I$. Suppose that $A$ is not essential in $R$. Then there exists a nonzero ideal $J$ 
of $R$ such that $A \cap J=\{0\}$ and $(A+J) / A$ is a nonzero ideal of a simple ring $R / A$. Thus $A+J=R$. This means that $A \oplus J=R$, which is a contradiction. So $A$ is essential in $R$. According to Proposition 2.2 and Theorem 3.4 there exists an embedding of rings $h: R \rightarrow \operatorname{ME}(A)$ such that $h \mid A=\mathrm{id}_{A}$. From this it follows that $h(R) / A \cong R / A$ and $A \cong h(R) / A$. This shows that $A$ is embeddable in $\operatorname{ME}(A) / A$.

Conversely, suppose that $A$ is embeddable in $\operatorname{ME}(A) / A$. Then there exists a subring $S$ of $\operatorname{ME}(A)$ such that $A \triangleleft S$ and $S / A \cong A$. But $a_{\operatorname{ME}(A)}(A)=\{0\}$ by Proposition 2.1. Thus $a_{S}(A)=\{0\}$ and, again by Proposition 2.1, $A$ is essential in $S$. Hence, there does not exist $I \triangleleft S$ such that $A \oplus I=S$. It follows that $A$ does not satisfy a Gardner condition.

In [4] it was proved that if a simple ring $A=A^{2}$ satisfies a Gardner condition then the lower radical determined by the class of all rings isomorphic to $A$ is an atom in the lattice of all radicals. By the above remark and Proposition 4.8 we obtain the following theorem.

THEOREM 4.9. If a simple ring $A=A^{2}$ is not embeddable in a ring $\mathrm{ME}(A) / A$ then the lower radical determined by the class of all rings isomorphic to $A$ is an atom in the lattice of all radicals.

\section{References}

[1] K. I. Beidar, 'Atoms in the "lattice" of radicals', Mat. Issled. 85 (1985), 21-31 (in Russian).

[2] K. I. Beidar, 'On essential extensions, maximal essential extensions and iterated maximal essential extensions in radical theory', in: Theory of Radicals (Szekszard, 1991), Colloq. Math. Soc. János. Bolyai, 61 (North-Holland, Amsterdam, 1993), pp. 17-26.

[3] J. F. Flanigan, 'On the ideal and radical embedding of algebras I. Extreme embeddings', J. Algebra 50(1) (1978), 153-174.

[4] B. J. Gardner, 'Simple rings whose lower radical are atoms', Acta. Math. Hungar. 43 (1984), $131-135$.

[5] W. G. Leavitt and L. C. A. Van Leeuwen, 'Multiplier algebras and minimal embeddability', Publ. Math. Debrecen 29 (1982), 95-99.

[6] E. R. Puczyłowski, 'On essential extensions of rings', Bull. Aust. Math. Soc. 35 (1987), 379-386.

\section{R. R. ANDRUSZKIEWICZ, Institute of Mathematics, University of Białystok, 15-267, Białystok, Akademicka 2, Poland e-mail: randrusz@math.uwb.edu.pl}

